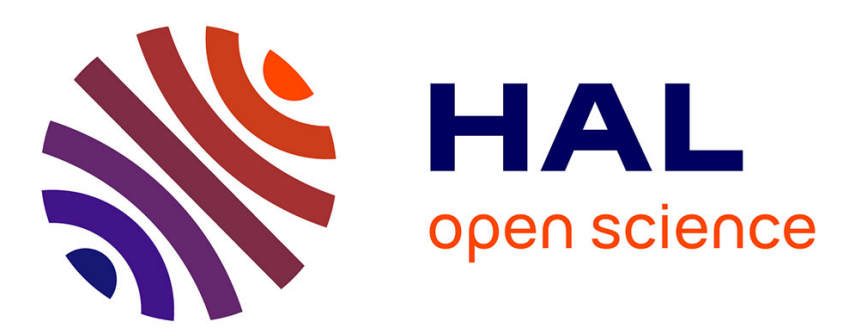

\title{
Three-Dimensional Motion Tracking of Coronary Arteries in Biplane Cineangiogram
}

Guy Shechter, Frédéric Devernay, Ève Coste-Manière, Arshed Quyyumi, Elliott R. Mcveigh

\section{- To cite this version:}

Guy Shechter, Frédéric Devernay, Ève Coste-Manière, Arshed Quyyumi, Elliott R. Mcveigh. ThreeDimensional Motion Tracking of Coronary Arteries in Biplane Cineangiogram. IEEE Transactions on Medical Imaging, 2003, 22 (4), pp.493-503. 10.1109/TMI.2003.809090 . inria-00262117

\section{HAL Id: inria-00262117 https://hal.inria.fr/inria-00262117}

Submitted on 11 Mar 2008

HAL is a multi-disciplinary open access archive for the deposit and dissemination of scientific research documents, whether they are published or not. The documents may come from teaching and research institutions in France or abroad, or from public or private research centers.
L'archive ouverte pluridisciplinaire HAL, est destinée au dépôt et à la diffusion de documents scientifiques de niveau recherche, publiés ou non, émanant des établissements d'enseignement et de recherche français ou étrangers, des laboratoires publics ou privés. 


\title{
Three-Dimensional Motion Tracking of Coronary Arteries in Biplane Cineangiograms
}

\author{
Guy Shechter*, Frédéric Devernay, Eve Coste-Manière, Arshed Quyyumi, and Elliot R. McVeigh
}

\begin{abstract}
A three-dimensional (3-D) method for tracking the coronary arteries through a temporal sequence of biplane X-ray angiography images is presented. A 3-D centerline model of the coronary vasculature is reconstructed from a biplane image pair at one time frame, and its motion is tracked using a coarse-to-fine hierarchy of motion models. Three-dimensional constraints on the length of the arteries and on the spatial regularity of the motion field are used to overcome limitations of classical two-dimensional vessel tracking methods, such as tracking vessels through projective occlusions. This algorithm was clinically validated in five patients by tracking the motion of the left coronary tree over one cardiac cycle. The root mean square reprojection errors were found to be submillimeter in $93 \%(54 / 58)$ of the image pairs. The performance of the tracking algorithm was quantified in three dimensions using a deforming vascular phantom. RMS 3-D distance errors were computed between centerline models tracked in the X-ray images and gold-standard centerline models of the phantom generated from a gated 3-D magnetic resonance image acquisition. The mean error was $0.69( \pm 0.06) \mathrm{mm}$ over eight temporal phases and four different biplane orientations.
\end{abstract}

Index Terms-Angiocardiography, geometric modeling, stereo vision, tracking.

\section{INTRODUCTION}

$\mathbf{X}$ -RAY coronary angiography is the gold-standard imaging technique for visualizing the morphology and motion of the coronary arteries. A pair of images, obtained using a biplane angiography system, can be used to reconstruct the three-dimensional (3-D) structure of the vascular tree [1]-[6]. These 3-D coronary reconstructions at multiple phases of the cardiac cycle have been used to describe the motion and function of the left ventricle epicardial surface [7]-[9], to quantify vessel lengths,

Manuscript received April 17, 2002; revised November 21, 2002. This work was supported by the French Ministry of Research and the Ministry of Economy, Finance, and Industry under the Telemedicine Project of the Réseau National Technologies pour la Santé. The work of G. Shechter was supported by the National Institutes of Health (NIH) under a Pre-IRTA Fellowship. The Associate Editor responsible for coordinating the review of this paper and recommending its publication was M. Sonka. Asterisk indicates corresponding author.

*G. Shechter is with the Department of Biomedical Engineering, Johns Hopkins University, Baltimore, MD 21205 USA, the Chir Group, INRIA, Sophia Antipolis, 06902 France, and the Laboratory of Cardiac Energetics, NHLBI, National Institutes of Health, DHHS, Bethesda, MD 20892 USA (e-mail: guy@jhu.edu).

F. Devernay and E. Coste-Manière are with the Chir Group, INRIA, Sophia Antipolis, 06902 France.

A. Quyyumi is with the Division of Cardiology, Emory University, Atlanta, GA 30322 USA.

E. R. McVeigh is with the Department of Biomedical Engineering, Johns Hopkins University, Baltimore, MD 21205 USA and the Laboratory of Cardiac Energetics, NHLBI, National Institutes of Health, DHHS, Bethesda, MD 20892 USA.

Digital Object Identifier 10.1109/TMI.2003.809090 volume, and flow [1], [10], and to quantify stenosis morphology [2].

The classical method for recovering the motion of the coronaries is to track the motion of the arterial centerlines in the two-dimensional (2-D) projection images. For each cardiac phase, a 3-D coronary tree is independently reconstructed. Optical flow [11], binary image elastic registration [12], Kalman snakes [13], and local space search and graph minimization techniques [14] are some of the methods that have been proposed for 2-D vessel tracking. However, tracking the vessels in the projection image space has significant limitations. Multiple vessel overlap is common due to the projective nature of the imaging modality. Correctly tracking arteries through these regions is a difficult problem, which can only be solved with additional knowledge and regularizing constraints. Yet, the use of true 3-D constraints on the shape and length of the coronaries, as well as on the spatial regularity of the 3-D motion of the coronary tree, is impossible when operating in the 2-D image space.

Making the transition to tracking the motion of the arteries in 3-D, Ruan [15] proposed combining optical flow derived displacement information from two projection images to displace an existing 3-D coronary tree model. A deformable models approach for segmenting objects in a stereo image pair using parametric curves was described by Bascle [16] and applied to segmenting the coronary arteries with 3-D B-spline curves by Radeva [17] and Cañero [18]. Toledo [19] extended this work into the temporal dimension by tracking the motion of the 3-D curves by allowing each B-spline control point to move independently in 3-D. An energy minimization scheme, using image derived forces and external constraint forces, is used to update the position of the control points. Due to the large number of degrees of freedom (DOFs) associated with this formulation, a Kalman filter, initialized with the average 3-D structure and motion of the coronary tree defined over a patient population, is used to constrain the procedure. Other 3-D knowledge based methods include the use of eigensnakes [20], and Fourier descriptors [21] to track the arteries; recently, an active contour method based on a biomechanical model was used to track a pacemaker lead in biplane images [22].

This paper presents a novel 3-D method for tracking the motion of the coronary arteries through a biplane cineangiography image sequence. A 3-D model of the coronary artery centerlines is reconstructed at one time frame, and represented as an ensemble of 3-D B-spline curves. An energy minimization problem within a registration framework is formulated to deform the coronary tree consistently with the angiogram image pairs at later time frames. Using 3-D constraints on the length 
changes of the coronary arteries and on the spatial regularity of their motion, an hierarchical set of coarse-to-fine 3-D motion models is used to recover the temporal evolution of the coronary tree.

A description of the methods used for image preprocessing, and for reconstruction of a 3-D coronary model from a biplane image pair is presented first. The motion models and the motion tracking method is presented in Sections II-D and Section II-E. The performance of this method is quantified in a phantom and using clinical angiograms, and results are presented in Section III. Further analysis and remarks are offered in Sections IV and Section V. A previous version of this work can be found in [23].

\section{METHODS}

\section{A. Imaging Protocol}

Biplane images (Philips BH5000, 15 frames/s, $512 \times 512$ pixels) of the left coronary tree were acquired for five patients enrolled in a clinical research protocol approved by the NIH safety review board. The patients were asked to suspend their breathing immediately prior to the contrast injection. The orientation of the imaging planes was unconstrained and selected to maximize separation of the arteries on the projection images. Later, the imaging system was reoriented to the same configurations used during the acquisition of patient data, and images of a calibration grid and a phantom were acquired. A calibration grid of radio-opaque beads was imaged while affixed to the image intensifier (II); the plastic rectangular phantom containing nine metal beads was imaged at a location approximating the patient's heart.

\section{B. Geometric Distortion Correction and Validation of Imaging Geometry}

Images acquired on an X-ray system equipped with a conventional II suffer from two independent geometric distortions: 1) the curved face of the II generates a pincushion distortion, which is observable as a stretching in the periphery of images; and 2) the interaction of the Earth's magnetic field with the electrons in the II generates an orientation dependent S-shaped distortion. Making accurate quantitative 3-D models of coronary morphology and motion depends on eliminating these artifacts.

A detailed explanation of the two step distortion correction method used for this study can be found in [23]. In summary, high order geometric distortion is removed by least-squares fitting of fifth degree polynomials to model the observed distortion of the calibration grid. Corrected images are synthesized by bilinear interpolation of the distorted images using the recovered polynomial functions. However, because the undistorted image of the calibration grid is computed from the central region of the distorted image, low-order distortion which affects the entire image is not recoverable in this step.

Reconstruction of the phantom, and comparison to its known geometry, is used to validate the imaging geometry, as recorded by the angiography system at the time of imaging, and to recover any low-order image distortion. A nonlinear least squares method is used to find the in-plane rotations of the two imaging planes and the 2-D in-plane translation of one imaging plane.

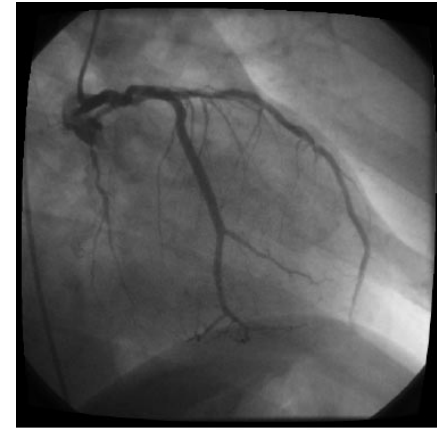

(a)

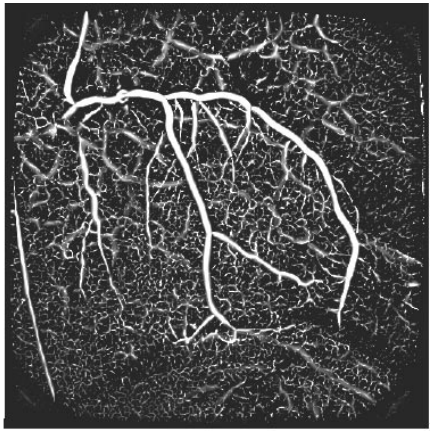

(b)
Fig. 1. (a) Example projection image of the left coronary tree (45 $\left.{ }^{\circ} \mathrm{RAO}\right)$ and (b) its corresponding maximum multiscale response map. The multiscale analysis retains rectilinear structures and removes planar structures such as the diaphragm and ribs.

The recovered parameters are used to correct the projection matrices for patient studies which used the same imaging arm orientation as a given phantom study.

\section{Reconstruction of a 3-D Coronary Tree}

The 3-D motion tracking method presented in this paper requires an a priori 3-D model of the coronary tree. For each patient, this model is reconstructed from one pair of biplane images at end-diastole using the method described in [6]. The angiogram images are first processed with a set of multiscale filters which detect the centerlines of rectilinear structures [24]. The highest response over the set of filters is stored as the maximum response map for that image, which, by thresholding, provides a noisy segmentation of the coronary centerlines (Fig. 1). A semi-interactive tool is then used to segment the arterial centerlines on the projection images, using the maximum multiscale response map to constrain the procedure.

The 2-D centerlines are represented as cubic B-splines, and are hierarchically organized. A dynamic programming graph search strategy defines point to point correspondences along the lengths of the drawn arteries based on the epipolar constraint. Finally, a discrete 3-D model of the coronary centerlines is reconstructed by computing the least-squares intersections of rays connecting matched projection points with their X-ray source (Fig. 2).

To reduce storage and computational requirements for tracking the motion of the coronaries, the discrete representation of the tree is converted to a parametric representation. The use of B-spline basis functions provides a continuous and compact representation both in 3-D and in the projected 2-D images, and provides intrinsic smoothness to the curves [25]. The discrete coronary tree is first decomposed into a set of arterial segments $\left\{A_{0}, A_{1}, \ldots\right\}$. Each segment, $A_{k}$, is defined as the ordered set of points describing a unique part of the coronary tree so that: 1) the segment includes and is terminated at both ends by a bifurcation point; or 2) is terminated by a bifurcation point at one end, and at the other end, by a leaf or root node of the tree. An approximating parametric curve $\mathbf{C}_{k}(u) \in \mathbb{R}^{3}$, is fit to each $A_{k}=\left\{\mathbf{q}_{0}, \mathbf{q}_{1}, \ldots, \mathbf{q}_{m_{k}}\right\} \in \mathbb{R}^{3}$ using a chord length parameterization so that

$$
\left\{\mathbf{C}_{k}(u) \mid u \in[0,1]\right\} \approx A_{k} .
$$




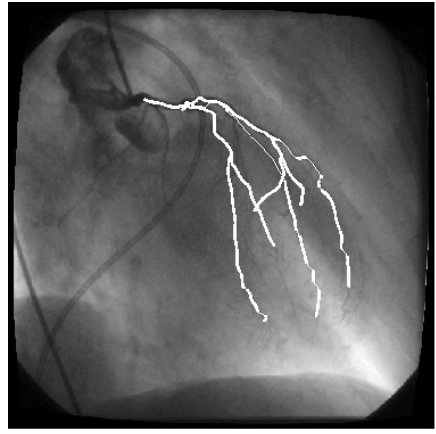

(a)

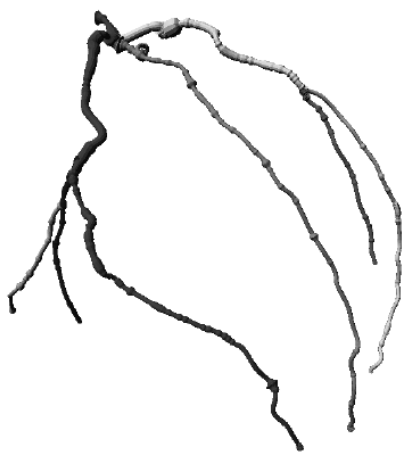

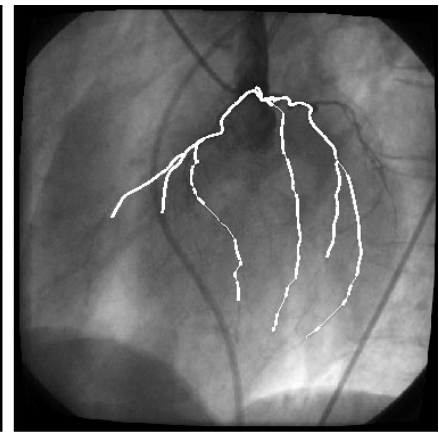

(b)

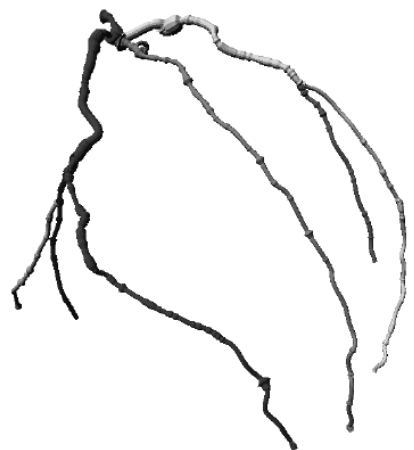

(c)

Fig. 2. (a) RAO and (b) LAO projections of the left coronary tree at end diastole. In (c), a wide-eyed stereogram of the reconstructed arteries, where the vessel diameters reflect the scale with the largest response in the artery detection step.

Specifically, $\mathbf{C}_{k}(u)$ is a 3-D B-spline curve defined as

$$
\mathbf{C}_{k}(u)=\sum_{i=0}^{n_{k}-1} B_{i, p}(u) \mathbf{V}_{i}^{k}
$$

where the $\left\{\mathbf{V}_{i}^{k}\right\} \in \mathbb{R}^{3}$ are the $n_{k}$ control points, and the $\left\{B_{i, p}\right\}$ are the $p$ th-degree $\mathrm{B}$-spline basis functions. Using the length $(m+1)$ nonperiodic and nonuniform knot vector $U_{k}$, where $m=n_{k}+p+1$, and

$$
U_{k}=\{\underbrace{0, \ldots, 0}_{p+1}, u_{p+1}, \ldots, u_{m-p-1}, \underbrace{1, \ldots, 1}_{p+1}\}
$$

the values at the ends of the curve are constrained so that

$$
\begin{aligned}
& \mathbf{C}_{k}(0)=\mathbf{V}_{0}^{k}=\left(\mathbf{q}_{0} \in A_{k}\right) \\
& \mathbf{C}_{k}(1)=\mathbf{V}_{n_{k}}^{k}=\left(\mathbf{q}_{m_{k}} \in A_{k}\right) .
\end{aligned}
$$

This strategy allows $C^{0}$ continuity to be maintained between a parent artery, $A_{k}$, and a child artery, $A_{j}$, by forcing them to share a control point

$$
\mathbf{C}_{k}(1)=\mathbf{C}_{j}(0) \quad \text { if } \quad \mathbf{V}_{n_{k}}^{k}=\mathbf{V}_{0}^{j} .
$$

In practice, cubic B-spline basis functions (degree $3, C^{2}$ continuity) are used for all artery segments, and $C^{0}$ continuity is maintained across the bifurcations.

\section{Motion Models}

The goal of the motion tracking algorithm is to recover the set of transformations $\left\{M^{t}: \mathbb{R}^{3} \rightarrow \mathbb{R}^{3}\right\}$, which map any point $\mathbf{q}$ on the coronary tree at time $t_{0}$, to the point's position at time $t_{0}+t$,

$$
\mathbf{q}^{t}=M^{t}(\mathbf{q})
$$

A hierarchical coarse-to-fine approach for recovering the transformation $M^{t}$ is used [26]. In this section, a description of the three motion models used to recover the motion of the coronary arteries is presented. From global to local, the motion models are a global 3-D rigid body transformation, a global 3-D affine transformation, and a local 3-D tensor product B-spline (B-solid [27]) transformation.

1) Three-Dimensional Rigid Body Transformation: The class of 3-D rigid body transformations describes motion of a nondeforming object in $\mathbb{R}^{3}$ using six DOFs: three angles of rotation, and three translation parameters. The transformation of a point $\mathbf{q}$ at time $t_{0}$ to its position $\mathbf{q}^{t}$ at time $t_{0}+t$ can be written as

$$
M_{R}^{t}(\mathbf{q})=\left[\begin{array}{lll|r}
R_{t} & & T_{t} \\
\hline 0 & 0 & 0 & 1
\end{array}\right]\left[\begin{array}{c}
\mathbf{q} \\
\overline{1}
\end{array}\right]
$$

where in $\mathbb{R}^{3}, R_{t}$ is a $3 \times 3$ rotation matrix with Euler angles $\left(\theta_{t}\right.$, $\left.\phi_{t}, \psi_{t}\right)$ and $T_{t}=\left(\Delta x_{t}, \Delta y_{t}, \Delta z_{t}\right)^{T}$ is a 3-D translation vector. The use of Euler angles is justified because the magnitude of the rotations are expected to be small. Since B-splines are invariant under rigid transformation, the rigid body transformation functional of the curve $\mathbf{C}(u)$ from (2) is expressed as

$$
M_{R}^{t}[\mathbf{C}]=\sum_{i=0}^{n-1} B_{i, p}(u) M_{R}^{t}\left(\mathbf{V}_{i}\right)
$$

2) Three-Dimensional Affine Transformation: The class of 3-D affine transformations describes motion of a nonrigid object in $\mathbb{R}^{3}$ using 12 DOFs. The transformation of a point $q$ at time $t_{0}$ to its position $\mathbf{q}^{t}$ at time $t_{0}+t$ can be written as

$$
M_{A}^{t}(\mathbf{q})=\left[\begin{array}{lll|c} 
& & & T_{t} \\
& & & \\
\hline 0 & 0 & 0 & 1
\end{array}\right]\left[\begin{array}{c}
\mathbf{q} \\
\overline{1}
\end{array}\right]
$$

where in $\mathbb{R}^{3}, A_{t}$ is a $3 \times 3$ matrix with nine free parameters $\left(a_{11}, a_{12}, a_{13}, \ldots, a_{33}\right)$ and $T_{t}=\left(\Delta x_{t}, \Delta y_{t}, \Delta z_{t}\right)^{T}$ is a 3-D translation vector. The matrix $A_{t}$ can be decomposed into three rotations, three scale factors, and three shears. Since B-splines are invariant under affine transformation, the 3-D affine transformation functional of the curve $\mathbf{C}(u)$ from (2) is expressed as

$$
M_{A}^{t}[\mathbf{C}]=\sum_{i=0}^{n-1} B_{i, p}(u) M_{A}^{t}\left(\mathbf{V}_{i}\right)
$$

3) B-solid Transformation: A B-solid transformation is used to model local deformation of the coronary arteries. The B-solid is the dimension three analog of the one-dimensional B-spline curve, and the 2-D B-spline tensor product surface. For a point on the coronary tree at $t_{0}$, the B-solid $D^{t}: \mathbb{R}^{3} \rightarrow \mathbb{R}^{3}$ gives the 3-D displacement vector to that point's position at 
time $t_{0}+t$. Given a point $\mathbf{q}=\left(q_{x}, q_{y}, q_{z}\right) \in \mathbb{R}^{3}$ at time $t_{0}$, its displacement to time $t_{0}+t$ is expressed as

$$
D^{t}(\mathbf{q})=\sum_{i=0}^{n_{i}-1} \sum_{j=0}^{n_{j}-1} \sum_{k=0}^{n_{k}-1} B_{i, p}\left(q_{x}\right) B_{j, p}\left(q_{y}\right) B_{k, p}\left(q_{z}\right) \mathbf{S}_{i j k}^{t}
$$

where the $\left\{\mathbf{S}_{i j k}^{t}\right\} \in \mathbb{R}^{3}$ are the $n_{i} \times n_{j} \times n_{k}$ control points, and the $\{B \cdot, p\}$ are the $p$ th-degree B-spline basis functions. The control point density is directly proportional to the amount of local deformation that can be represented by the B-solid. By using cubic B-spline basis functions $(p=3), C^{2}$ continuity of the deformation field is maintained over the B-solid volume. Each B-spline basis function is defined on uniform knot vectors aligned with the patient's superior-inferior, right-left, and anterior-posterior axes. The B-solid is defined over the volume of the 3-D coronary tree, with a border on each side of $10 \%$ of the range in that dimension. Finally, the B-solid transformation of a point $\mathbf{q}$ at time $t_{0}$ to its position $\mathbf{q}^{t}$ at time $t_{0}+t$ is expressed as

$$
M_{B}^{t}(\mathbf{q})=\mathbf{q}+D^{t}(\mathbf{q}) \text {. }
$$

The B-solid transformation functional for the curve $\mathbf{C}(u)$ from (2) is expressed as

$$
M_{B}^{t}[\mathbf{C}]=\sum_{i=0}^{n-1} B_{i, p}(u) M_{B}^{t}\left(\mathbf{V}_{i}\right)
$$

in order to simplify the motion recovery procedure. However, the B-solid transformation of the control points of the coronary artery B-splines is not strictly the same as the B-solid transformation of evaluations of the coronary artery B-splines. The use of the formulation in (14) is justified when the control point density of the B-solid is much smaller than the control point density of the coronary artery B-spline curve. Quantitative analysis supporting the use of this formulation is presented in Section III.

\section{E. Motion Tracking}

A registration framework is adopted to recover the set of transformations $\left\{M^{t}: \mathbb{R}^{3} \rightarrow \mathbb{R}^{3}\right\}$ which deform and orient the 3 -D coronary tree reconstructed at time $t_{0}$, to its configuration at time $t_{0}+t$, consistent with the observed angiogram images at time $t_{0}+t$. A closed system is defined, composed of a 3-D coronary tree defined at time $t_{0}$, the motion transformation $M^{t}$, and a biplane angiogram image pair at time $t_{0}+t$. An energy measure describing the quality of the transformed coronary tree's fit to the angiogram images is expressed as

$$
E_{\text {total }}=-\omega_{1} E_{\text {external }}+\omega_{2} E_{\text {arteries }}+\omega_{3} E_{\mathrm{bsolid}}
$$

which is a weighted sum of an external angiographic image derived energy term, an internal arterial energy term, and when solving for the B-solid transformation, an internal B-solid energy term. The goal of the motion tracking procedure is to recover the "best" transformation, $M^{t}$, which minimizes the energy functional defined in (15) and nulls its derivative taken with respect to the transformation parameters. Analytical derivatives are computed and the solution is recovered with a Lagrangian explicit numerical scheme, a gradient descent method [16], [28].
To solve for $M^{t}$ at time $t_{0}+t$, the rigid transformation, $M_{R}^{t}$, is recovered first using a multiresolution approach. The maximum multiscale response map corresponding to the angiogram images at time $t_{0}+t$ is smoothed using a Gaussian kernel with a standard deviation of $\sigma_{R}$. When the procedure for recovery of $M_{R}^{t}$ converges at this resolution, the standard deviation of the smoothing kernel is halved, and the procedure continues iterating on a sharpened image. This procedure is repeated until the standard deviation of the smoothing kernel reaches a stopping condition.

The recovered rigid transformation $M_{R}^{t}$ is used to initialize the multiresolution search for the affine transformation, $M_{A}^{t}$. When solving for the affine transformation, all 12 parameters are treated as DOFs, which may cause the rotation and translation parameters to change from those recovered in the rigid tracking step. Similarly, $M_{A}^{t}$ is used to initialize the multiresolution recovery of the B-solid transformation, which, after convergence, is the desired final transformation $\left(M^{t}=M_{B}^{t}\right)$. Finally, $M^{t}$, the recovered transformation at time $t_{0}+t$, is used to initialize the search for $M^{t+1}$.

This multiresolution tracking strategy is combined with the set of hierarchical motion models, with increasing DOFs, in order to overcome the local minimum sensitivity of the gradient descent method.

1) External Energy: The external energy term is a measure of how well the transformed coronary arteries project onto the angiogram images. $E_{\text {external }}$ is calculated using a potential map, $R_{i}$, computed for the projection X-ray angiography image, $I_{i}$, using the multiscale response method described in Section II-C. The gradient descent method attracts the arterial model's projections into the valleys of the multiscale response map, without having to explicitly extract and label vessel centerlines in the angiogram images.

For a given motion functional, $M^{t}$, and a set of curves defining the 3-D coronary tree at time $t_{0},\left\{\mathbf{C}_{a}(u)\right\}$, the values of the potential field of the projected transformed curves are integrated along their 3-D arc-length and normalized by the length of the coronary tree and the number of projections

$$
\begin{aligned}
E_{\text {external }}= & \left(n_{p} \sum_{a=1}^{n_{a}} L\left[\hat{\mathbf{C}}_{a}\right]\right)^{-1} \\
& \sum_{i=1}^{n_{p}} \sum_{a=1}^{n_{a}} \int_{0}^{L\left[\hat{\mathbf{C}}_{a}\right]} R_{i}\left(x_{i}^{a}(s), y_{i}^{a}(s)\right) d s
\end{aligned}
$$

where $n_{p}$ is the number of projection images, $n_{a}$ is the number of arteries in the 3-D coronary tree model, and the transformed coronary B-spline curve $\hat{\mathbf{C}}_{a}=M^{t}\left[\mathbf{C}_{a}\right]$ is itself a 3-D B-spline curve with control points $\hat{\mathbf{V}}_{i}=M^{t}\left(\mathbf{V}_{i}\right)$ [see (9), (11), and (14)]. The functional $L$ calculates the arc-length of a 3-D B-spline curve defined over the parametric interval $[0,1]$ by evaluating

$$
L\left[\mathbf{C}_{a}\right]=\int_{0}^{1}\left\|\mathbf{C}_{a}^{\prime}(u)\right\| d u .
$$

Finally, $x_{i}^{a}(u)$ and $y_{i}^{a}(u)$ are the projection coordinates of $\hat{\mathbf{C}}_{a}$ on the $i$ th projection image. Given the $3 \times 4$ projection matrix, $\mathbf{P}^{i}$, which defines the projection of the 3 -D model coordinates to 
local image coordinates for image $I_{i}$, the projected transformed coronary 3-D B-spline can be written in homogeneous coordinates as

$$
\mathbf{P}^{i} M\left[\mathbf{C}_{a}\right]=\mathbf{P}^{i} \hat{\mathbf{C}}_{a}(u)=\sum_{j=0}^{n-1} B_{j, p}(u) \mathbf{P}^{i} \hat{\mathbf{V}}_{j} .
$$

The nonhomogeneous coordinates of the projected curve can be expressed as two nonuniform rational B-splines (NURBS)

$$
\begin{aligned}
& x_{i}(u)=\frac{\sum_{j=0}^{n-1} B_{j, p}(u)\left(P_{1,1}^{i} \hat{V}_{j}^{x}+P_{1,2}^{i} \hat{V}_{j}^{y}+P_{1,3}^{i} \hat{V}_{j}^{z}+P_{1,4}^{i}\right)}{\sum_{j=0}^{n-1} B_{j, p}(u)\left(P_{3,1}^{i} \hat{V}_{j}^{x}+P_{3,2}^{i} \hat{V}_{j}^{y}+P_{3,3}^{i} \hat{V}_{j}^{z}+P_{3,4}^{i}\right)} \\
& y_{i}(u)=\frac{\sum_{j=0}^{n-1} B_{j, p}(u)\left(P_{2,1}^{i} \hat{V}_{j}^{x}+P_{2,2}^{i} \hat{V}_{j}^{y}+P_{2,3}^{i} \hat{V}_{j}^{z}+P_{2,4}^{i}\right)}{\sum_{j=0}^{n-1} B_{j, p}(u)\left(P_{3,1}^{i} \hat{V}_{j}^{x}+P_{3,2}^{i} \hat{V}_{j}^{y}+P_{3,3}^{i} \hat{V}_{j}^{z}+P_{3,4}^{i}\right)}
\end{aligned}
$$

Making the assumption that the length of a coronary artery changes minimally over the cardiac cycle, $L\left[\hat{\mathbf{C}}_{a}\right] \approx L\left[\mathbf{C}_{a}\right]$, [29], and writing the arc-length reparameterization of a B-spline curve as

$$
\begin{aligned}
s(\mathbf{C}, u) & =\int_{0}^{u}\left\|\mathbf{C}^{\prime}(\tilde{u})\right\| d \tilde{u} \\
\frac{d s(\mathbf{C}, u)}{d u} & =\left\|\mathbf{C}^{\prime}(u)\right\|
\end{aligned}
$$

allows (16) to be written as

$$
\begin{aligned}
E_{\text {external }}= & \left(n_{p} \sum_{a=1}^{n_{a}} L\left[\mathbf{C}_{a}\right]\right)^{-1} \\
& \sum_{i=1}^{n_{p}} \sum_{a=1}^{n_{a}} \int_{0}^{1} R_{i}\left(x_{i}^{a}(u), y_{i}^{a}(u)\right)\left\|\hat{\mathbf{C}}_{a}^{\prime}(u)\right\| d u .
\end{aligned}
$$

2) Arterial Energy: The internal arterial energy is a regularizing term that prevents large changes in the lengths of the arteries $\left\{\mathbf{C}_{a}(u)\right\}$ due to the transformation $M^{t}$

$$
E_{\text {internal }}=\sum_{a=1}^{n_{a}}\left(L\left[M^{t}\left[\mathbf{C}_{a}\right]\right]-L\left[\mathbf{C}_{a}\right]\right)^{2}
$$

where $n_{a}$ is the number of arteries in the 3-D coronary tree model, and $\mathrm{L}$ is the arc-length functional defined in (17).

3) B-solid Energy: The internal B-solid energy term is used to regularize the values at neighboring control points in the $\mathrm{B}$-spline grid. Therefore, the energy of the B-solid structure is defined as a 3-D Laplacian operator, and normalized by the number of control points in the B-solid

$$
\begin{aligned}
E_{\mathrm{bsolid}}= & \frac{1}{n_{i} n_{j} n_{k}} \sum_{e=0}^{n_{i}-1} \sum_{f=0}^{n_{j}-1} \sum_{g=0}^{n_{k}-1} \\
& \left(\sum_{i=-1}^{1} \sum_{j=-1}^{1} \sum_{k=-1}^{1} \mathbf{w}_{i, j, k} \cdot \mathbf{S}_{e+i, f+j, g+k}^{t}\right)^{2} .
\end{aligned}
$$

$w$ is a $9 \times 9 \times 9$ distance weighting function where $\mathbf{w}_{i, j, k}=$ $\left[\begin{array}{lll}w_{i, j, k} & w_{i, j, k} & w_{i, j, k}\end{array}\right]$ and

$$
w_{i, j, k}= \begin{cases}-\sum_{p=-1}^{1} \sum_{q=-1}^{1} & \\ \sum_{r=-1}^{1} \sqrt{|p|+|q|+|r|}, & \text { if }(i, j, k)=(0,0,0) \\ \sqrt{|i|+|j|+|k|}, & \text { if }(i, j, k) \neq(0,0,0) .\end{cases}
$$

\section{F. Validation of the Motion Tracking Algorithm}

After recovering a transformation $M^{t}$, an independent quantitative assessment of the motion tracking method's performance is desired. As described in the next sections, validation trials were performed on clinical angiogram images, and on a deforming vascular phantom.

1) Clinical Angiograms: The ultimate goal of the proposed algorithm is its application to tracking the coronary arteries in clinical angiograms. Unfortunately, because the true 3-D motion of the coronary tree is not known, the use of a 3-D metric is not possible. Instead, the error is quantified in the space of the 2-D projection images.

First, the projection angiogram images at time $t_{0}+t$ are segmented using the semi-interactive method described in Section II-C. This set of points representing the coronary centerlines for projection $I_{i}$ is labeled $F_{i}^{t}$. For the transformation $M^{t}$, the transformed coronary tree is computed and sampled in 3-D with an arc-length resolution of $1 \mathrm{~mm}$. Each point of the sampled transformed coronary tree is projected onto angiogram $I_{i}$ using the $3 \times 4$ projection matrix $\mathbf{P}_{i}$. For each projected point, a 2-D pixel distance is computed to the closest point in $F_{i}^{t}$. In order to standardize results between acquisitions, this distance is converted to millimeters using the known intensifier size and magnification factor for that acquisition. The reprojection error for $M^{t}$ is computed as the root mean square (RMS) of the 2-D norm distances in both projections.

In some patients, the distal coronary vessels disappear from the images, presumably due to extravascular myocardial compression during systole [30]. Therefore, any 3-D coronary point whose distance to the closest point in both projection images is greater than ten pixels is discarded. Visual inspection is used to confirm that this procedure does not eliminate points which belong to mistracked arteries.

2) Vascular Phantom: A deforming vascular phantom was constructed to facilitate a 3-D validation experiment. A tree of Tygon tubes (inner diameters between $0.58-3.18 \mathrm{~mm}$, total length $=34 \mathrm{~cm}$ ) was constructed, and filled with an iodinated contrast agent. The tubes were arranged around a compliant latex balloon which was inflated using a mechanical ventilator. Sample biplane X-ray images of the phantom are shown in Fig. 3.

The gold-standard reconstruction of the geometry and motion of this phantom was assessed with multislice gated magnetic resonance (MR) imaging. MR provided images with an in-plane spatial resolution of $0.4 \times 0.4 \mathrm{~mm}$, a slice thickness of $2 \mathrm{~mm}$, and a temporal resolution of $40 \mathrm{~ms}$ ( 25 cine phases/motion cycle). The gold-standard representation of the deforming 


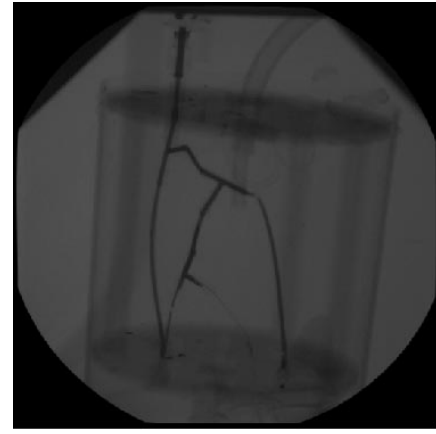

(a)

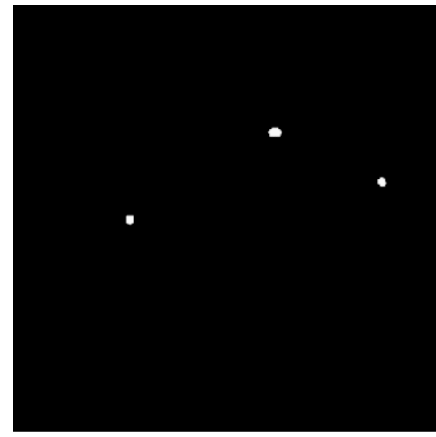

(c)

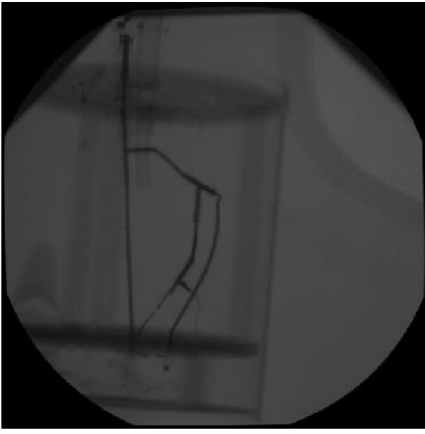

(b)

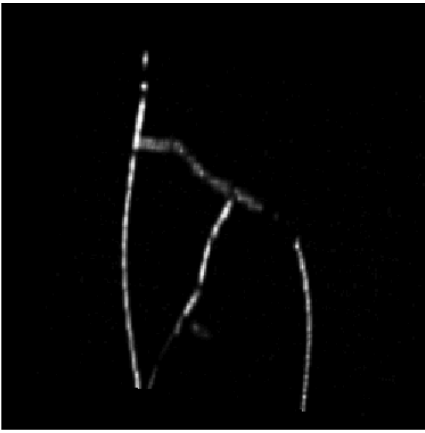

(d)
Fig. 3. (a) RAO and (b) LAO projections of the vascular phantom. In (c), one slice of the 3-D MR image volume of the phantom, and in (d) a maximum intensity projection of the MR images.

phantom was generated by manually delineating the centerlines in the MR images using a 3-D segmentation tool [31].

Because of the different temporal resolution and sampling frequency of the MR and X-ray acquisitions (15 frames/s), eight sets of X-ray/MR image groups that were most closely matched temporally were identified. The phantom was reconstructed from the first pair of X-ray images using the vascular reconstruction method described in Section II-C. A rigid registration procedure was used to align the centerline reconstructions obtained from X-ray and MR at the first motion phase, and the 3-D reconstruction error was computed by calculating the RMS of the closest point distances between these two modalities.

The motion of the reconstructed phantom was tracked through the remaining seven biplane image pairs. The resulting centerline models were then compared with the centerlines segmented from the MR images at the corresponding motion phase. The quality of the motion tracking algorithm was quantified by computing the RMS of the 3 -D closest point distances between the two modalities at each phase of the tracked motion. As a baseline error level, 3-D RMS errors were also measured between the untracked X-ray reconstruction from the first pair of X-ray images, and the MR reconstructions obtained at the other seven motion phases.

\section{RESULTS}

\section{A. Clinical Angiograms}

Three-dimensional models of the left coronary tree were reconstructed for five patients at end-diastole. The amount of the
TABLE I

CORONARY ARTERy SEgments RECONSTRUCTED FOR EACH PATIENT

\begin{tabular}{c|l}
\hline Patient & Reconstructed Coronary Segments \\
\hline 1 & LM, LAD, LCx, OM1 ${ }^{+}, \mathrm{OM} 2$ \\
2 & LM, LAD, D1 ${ }^{+}, \mathrm{LCx}, \mathrm{OM} 1$ \\
3 & LM, LAD, D1, D2, D3, LCx, OM1, OM2 \\
4 & LM, LAD, D1, D2, LCx \\
5 & LM, LAD, D1, D2, OM1, OM2 ${ }^{+}$ \\
\hline LM: Left main. \\
LCX: Left circumflex. \\
LAD: Left anterior descending. \\
D: Diagonal. \\
OM: Obtuse marginal. \\
(+ Indicates additional unnamed daughter branches.)
\end{tabular}

coronary vasculature that was reconstructed varied between patients depending on the pathological state of the arteries, and the ability to distinguish corresponding arterial segments in the given projection image pairs. A list of the coronaries reconstructed for each patient is provided in Table I.

The approximating 3-D B-spline curve representation of the coronaries was constructed with knots spaced at 2-mm intervals along the arc-length of the arteries. The maximum 3-D distance error from the approximating B-spline to the discrete points was $0.6 \mathrm{~mm}$ in the five reconstructions. The static 3 -D reconstruction was reprojected onto the images and the RMS reprojection error was calculated using the method described in Section II-F1. For each of the five patients, the reconstruction's reprojection RMS error was $0.2 \mathrm{~mm}$. This error can be partially attributed to the semi-interactive arterial segmentation step where the centerline positions are rounded to the nearest whole pixel.

Motion tracking was performed over one cardiac cycle, which ranged between 9 and 15 frames in the five data sets. The mean displacement ( \pm one standard deviation) of the coronary tree between successive image frames was $5.0( \pm 4.0), 5.2( \pm 3.1)$, $5.5( \pm 2.8), 7.1( \pm 4.4)$, and $7.4( \pm 4.3)$ pixels. The first iteration for each motion model operated on the potential map smoothed using a Gaussian kernel with a standard deviation, $\sigma$, of 4 pixels; the procedure was terminated when $\sigma=0.5$ pixels. Two of the patients required an additional preiteration of the rigid motion model with a smoothed potential map using $\sigma=8$ in order to capture the rapid motion of their arteries.

An $8 \times 8 \times 8$ control point grid was used for the B-solid motion model in all patients. For the rigid motion model, the coefficients of (15) were: $\omega_{1}=1, \omega_{2}=0$, and $\omega_{3}=0$. For the affine motion model, the coefficients were: $\omega_{1}=1, \omega_{2}=0.05$, and $\omega_{3}=0$. For the B-solid motion model, the coefficients were: $\omega_{1}=1, \omega_{2}=0.001$, and $\omega_{3}=0.0001$.

Tracking results for one of the patients are presented in Fig. 4. The recovered motion models are applied to the 3-D coronary tree reconstructed at end-diastole, and the results are reprojected on the angiogram images. The biplane image pairs correspond to images during the atrial contraction (top row), during the ventricular contraction (middle row), and at the next end-diastolic phase (bottom row).

Table II summarizes the number of iterations required for interframe convergence of each motion model for the five datasets. 

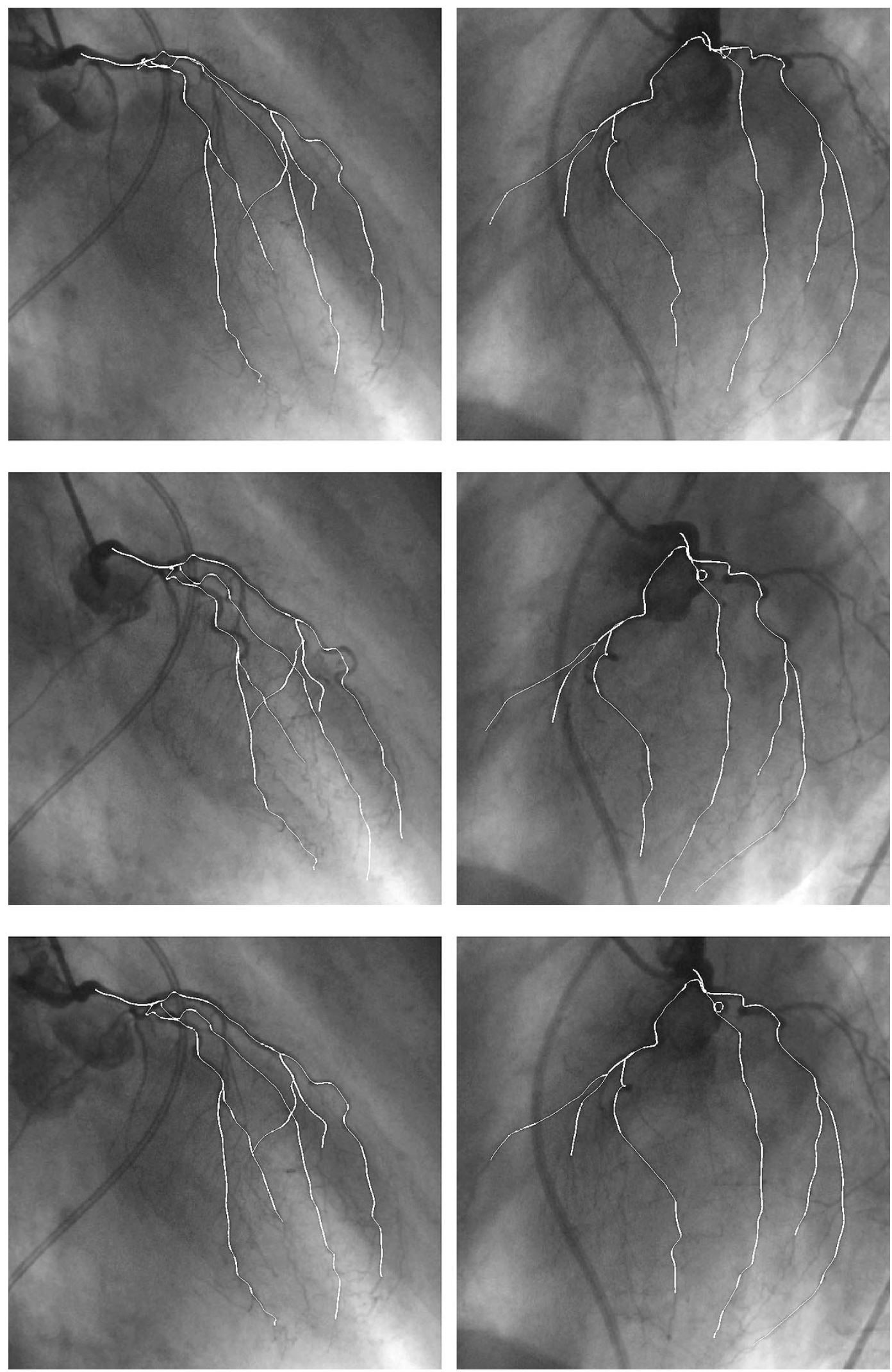

Fig. 4. Tracking results for one patient over one cardiac cycle. The biplane image pairs correspond to atrial contraction (top row), systole (middle row), and end-diastole (bottom row).

The increased number of iterations required for the rigid motion model in patients 3 and 5 correspond to the extra iteration with the larger spatial smoothing kernel of eight pixels described pre- viously. Fig. 5 shows the system energy as a function of the iteration number for five frames in one patient. The largest drops in the system energy occur during the initial rigid registration 
TABLE II

NUMBER OF ITERATIONS REQUIRED FOR INTERFRAME CONVERGENCE OF THE Motion Tracking Algorithm In Five Patient Datasets

\begin{tabular}{c|c|c|c|c}
\hline Patient & Tracked & \multicolumn{3}{|c}{ Mean Number of Iterations/Frame } \\
Number & Frames & Rigid & Affine & B-solid \\
\hline 1 & 13 & $60 \pm 14$ & $75 \pm 30$ & $115 \pm 28$ \\
2 & 12 & $57 \pm 20$ & $79 \pm 24$ & $136 \pm 59$ \\
3 & 15 & $99 \pm 33$ & $106 \pm 37$ & $127 \pm 73$ \\
4 & 10 & $72 \pm 19$ & $99 \pm 22$ & $142 \pm 34$ \\
5 & 14 & $91 \pm 20$ & $86 \pm 25$ & $119 \pm 31$
\end{tabular}

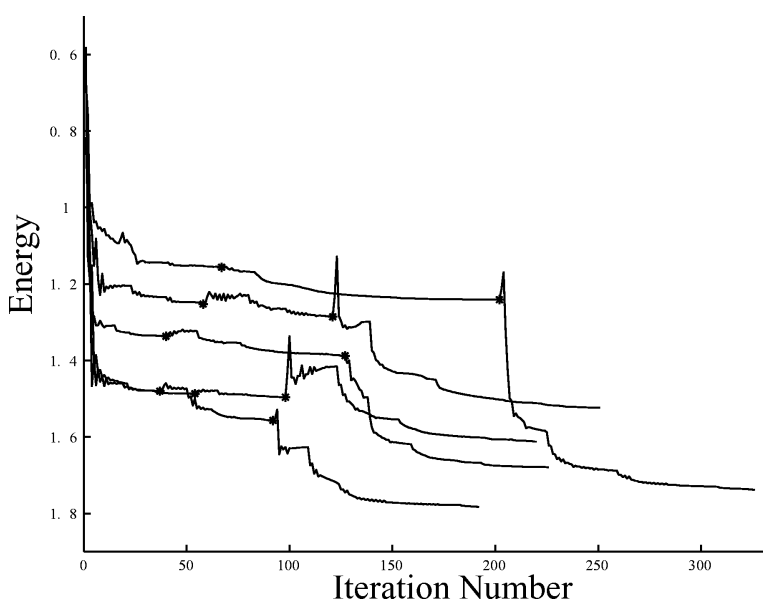

Fig. 5. Convergence plots for five interframe motion tracking steps in one clinical coronary angiogram dataset. Two stars on each curve indicate the transition between the rigid and affine motion tracking, and between the affine and B-solid motion tracking steps. The energy increases observed at the transitions between the different motion models is due to the larger spatial smoothing used for the initial recovery phase of each motion model as part of the multiresolution tracking strategy.

steps and during the B-solid tracking procedure. The energy increases observed at the transitions between the different motion models is due to the larger spatial smoothing used for the initial recovery phase of each motion model as part of the multiresolution tracking strategy (see Section II-E).

The RMS reprojection errors for the tracked arteries of the five patients are presented in Fig. 6. Because of the different heart rates, the five patients' results are rescaled temporally and plotted over one cardiac cycle. Submillimeter RMS errors were observed in 93\% (54/58) of the tracked biplane image pairs. In Fig. 7, the RMS reprojection error for one patient is plotted together with the average magnitude of 3-D displacements from end-diastole of sampled points on the coronary tree.

Following recovery of the motion transformations for the five data sets, the validity of (14) was assessed. In the first case, the 3 -D B-spline coronary tree was sampled at time $t_{0}$, and the samples were subsequently transformed to time $t_{0}+t$. In the second case, the control points of the 3-D B-spline coronary tree were transformed from time $t_{0}$ to time $t_{0}+t$ and then sampled. The 3 -D displacement errors between the two cases were quantified on a point by point basis, and were under $0.1 \mathrm{~mm}$ in all patients throughout the cardiac cycle. The results are not unexpected as the density of the control points of the 3-D coronary

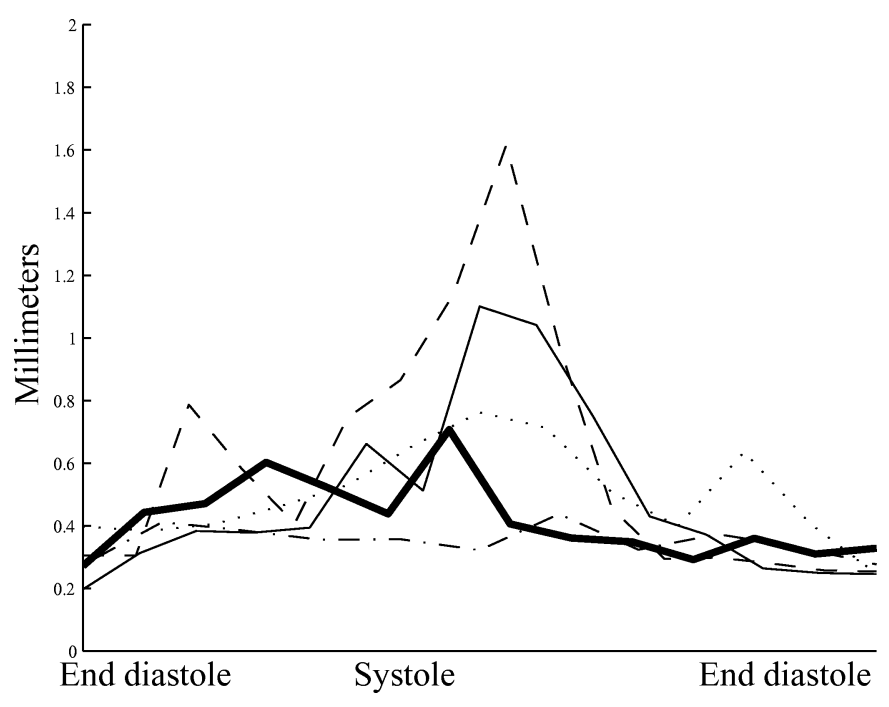

Fig. 6. The RMS reprojection errors following tracking through one cardiac cycle in five patient data sets.

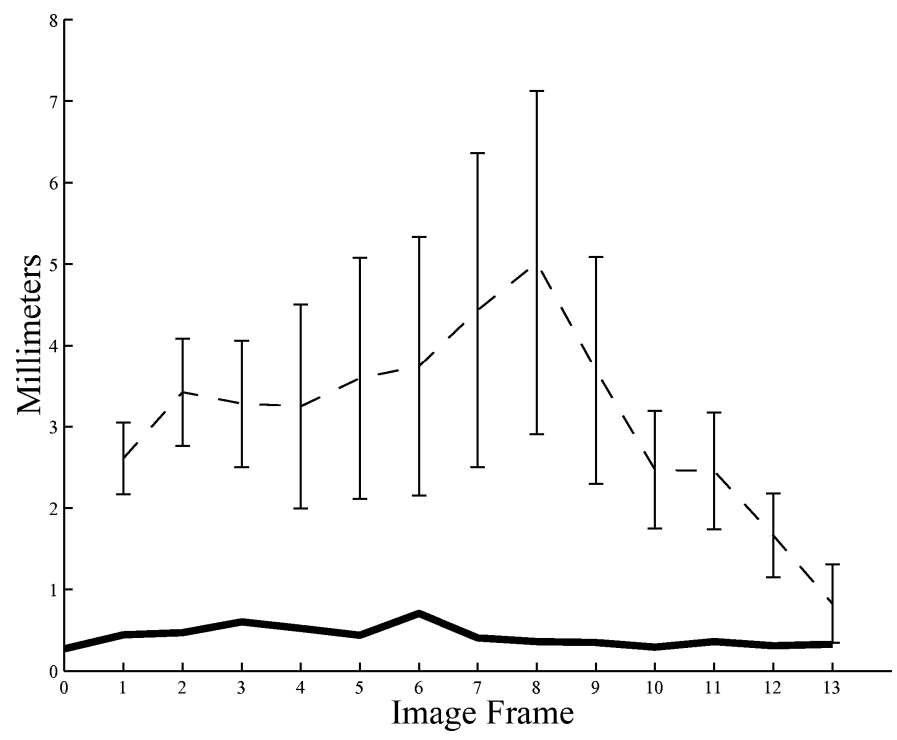

Fig. 7. In one patient, the mean 3-D displacement (dashed line) from end-diastole of points on the coronary tree are shown in comparison to the measured RMS reprojection error (solid line). Error bars show one standard deviation of the 3-D displacements. The first image frame represents end-diastole, and the 14 frames span one cardiac cycle.

B-splines are much higher (every $2 \mathrm{~mm}$ along the arc-length) than the B-solid control point density (between 10-20 mm).

These algorithms were implemented in MATLAB (Mathworks, Inc., Natick, MA) and the five data sets were processed on computers running Linux, with Pentium III processors operating between 0.75 and $1 \mathrm{GHz}$. The mean running time for motion recovery for one time frame was $155 \min (\sigma=58$ min). Reimplementation in a compiled language, with more efficient use of memory, is needed to reduce execution times for clinical application.

\section{B. Vascular Phantom}

The vascular phantom was reconstructed from a biplane image pair and rigidly aligned with the centerline model extracted from the MR images. This was repeated using four 


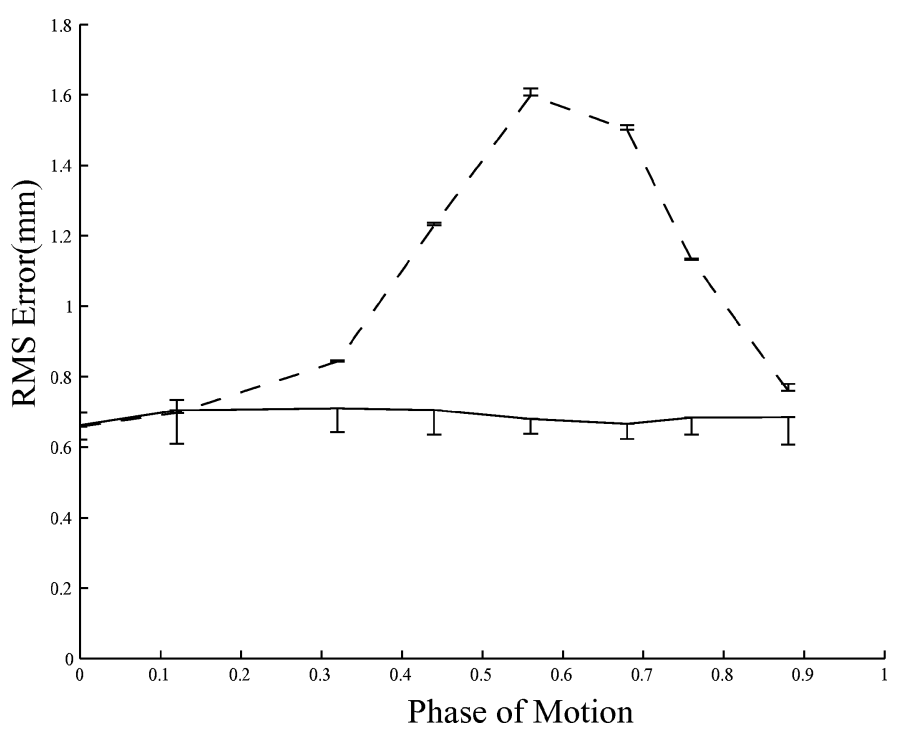

Fig. 8. The vascular phantom was reconstructed and tracked through seven pairs of biplane X-ray images. The solid line shows the 3-D RMS error between the tracked vascular phantom and the gold-standard centerline models obtained from MR imaging at each motion phase. As a baseline error level (dashed line), the 3-D RMS error between the untracked X-ray reconstruction, and the deforming gold-standard MR reconstructions was computed. The lines represent the mean (and one standard deviation) of results obtained in four independent acquisitions obtained with different biplane angular separations.

sets of biplane image pairs obtained with different imager orientations, which corresponded to typical angular separations used in clinical biplane coronary angiography. Over the four reconstructions, the mean ( \pm one standard deviation) 3-D RMS error between the X-ray and MR reconstructions was $0.66( \pm$ $0.04) \mathrm{mm}$. Reconstructions obtained from image pairs with less than $20^{\circ}$ separation in the LAO-RAO direction showed increasing 3-D RMS reconstruction errors; with a primary angle separation of $14^{\circ}$, the RMS error was $1.3 \mathrm{~mm}$.

The vascular phantom was tracked into seven biplane image pairs, using similar parameters to those used for the clinical angiograms, except that $\omega_{2}=0.005$ for the B-solid motion model, and the largest spatial smoothing kernel used was $\sigma=2$ pixels. The maximum 3-D displacement of the phantom during its periodic motion was $2.9 \mathrm{~mm}$, with the mean 3-D displacement reaching $1.5 \mathrm{~mm}$. The mean displacement $( \pm$ one standard deviation) of the phantom between successive image frames was $1.1( \pm 0.8)$ pixels.

The 3-D RMS error computed between the tracked centerlines and their corresponding centerline models extracted from the MR imaging dataset are shown in Fig. 8 (solid line). The motion tracking algorithm succeeds in keeping the mean 3-D RMS error at $0.69( \pm 0.06) \mathrm{mm}$ over the four biplane reconstructions and over all tracked time frames. In comparison, the 3-D RMS errors between the untracked X-ray reconstruction and the deforming gold-standard MR reconstructions varies between 0.66 and $1.60 \mathrm{~mm}$ (dashed line).

\section{DISCUSSION}

This paper demonstrates the success of a 3-D method for recovering the motion of the left coronary artery tree in biplane angiograms of patients with a history of severe cardiac disease, in- cluding myocardial infarcts and diffuse coronary artery disease. The algorithm's generality allows its performance in tracking the right coronary artery to be evaluated. However, combining the motion of the two arterial trees into one B-solid deformation field would be complicated by any respiratory and patient motion between the two contrast injections.

The experiment performed using the vascular phantom further validates the performance of the motion tracking algorithm by providing an analysis in 3-D. The measured 3-D RMS errors for the tracked models were similar to the 3-D reconstruction error, indicating that the algorithm performs exceptionally well, and that the residual errors may be due to variability in the manual segmentation of the centerlines from the MR images.

The coronary tree is treated as a whole, with no special emphasis given to vessel bifurcations. This obviates the difficulty of explicitly identifying bifurcations, which can be obscured by overlapping vessels. In some cases, the two daughter vessels emanating from a bifurcation may overlap each other in the projection image, causing the point of bifurcation to appear more distal than its true position.

Previously published 3-D coronary motion tracking methods [17], [18] have treated the position of each arteries' B-spline control points as independent variables to be optimized, and have constrained the solution space using a priori knowledge of coronary motion [19]. The method presented in this paper formulates the motion tracking problem as a warping of the space in $\mathbb{R}^{3}$ spanned by the 3-D coronary model. The variables being optimized are the parameters of the 3-D rigid body and affine transformations, and the control points of the B-solid. These, in turn, generate a motion field which moves the coronaries in a spatially coherent manner. Except for the assumption that the motion of the arteries varies smoothly over $\mathbb{R}^{3}$, no prior knowledge about the coronary anatomy or motion is required.

The motion of nearby arteries, in a 3-D sense, can be implicitly used to facilitate convergence to the correct solution. Consider the case of tracking an artery through a multivessel overlap in the projection images. In subsequent time frames, when the arteries separate in the projection images, one of two or more arteries must be chosen as the correct artery to follow. Tracking of a neighboring artery, unambiguously resolvable in the projection images, would act to constrain the tracking procedure of the first artery. The spatial regularity of the warping of $\mathbb{R}^{3}$, imposed by the B-solid, guides the selection of the correct postocclusion artery to track.

As described in Section II-E, the motion of the coronary arteries is recovered one frame at a time. The jagged reprojection error plots of Fig. 6 suggest that the recovered transformations do not capture the temporal regularity typical of physiological motion. An extension to the method presented in this paper calls for the implementation of temporal continuity of the rigid and affine motion models and the use of four-dimensional tensor product B-splines. However, temporal discontinuities are expected during the cardiac cycle, particularly between the systolic and diastolic phases. This knowledge would have to be embedded in the temporal regularizing functions, in order to be able to correctly capture rapid motion changes.

Motion tracking with temporal continuity will also provide a framework to overcome inconsistencies due to the nonsyn- 
chronous nature of some biplane imaging systems which acquire the two image planes in a temporally interleaved mode. In the current implementation, a given biplane image pair is assumed to be acquired synchronously, when there is in fact a 16-ms temporal offset between the images. Rapid motion may produce irreconcilable observations in the two views, which would interfere with convergence of the motion tracking procedure for that time frame. Using temporally continuous motion models will allow each image to be assigned its correct temporal index.

The RMS reprojection errors of the five patients show a characteristic pattern of increasing during systole and decreasing into diastolic relaxation. There are two factors which may contribute to this observation. First, the deformation of the coronary arteries over the cardiac cycle, especially in patients with highly tortuous vasculature, may be too localized to be adequately modeled using the $8 \times 8 \times 8$ control point grid used for the B-solid motion model in these experiments. This control point density was chosen based on experiments for recovery of myocardial motion from tagged magnetic resonance images [32]. However, the use of more dense control point grids was avoided because of the prohibitive computation times given the current implementation. In lieu of increasing the control point density, a deformable models method may be considered as a final step for capturing highly local shape changes.

A second explanation is linked to the observed disappearance of the distal parts of the coronary arteries during systole. Physiologically, extravascular compression of the intramyocardial arteries can lead to their collapse or to a reversal of flow in the coronaries during systole [30]. For example, in Fig. 4, distal coronary segments visible in the end-diastolic images (rows 1 and 3) disappear in the systolic images (row 2). The disappearance of vessels during part of the cardiac cycle might cause the 3-D coronary model to be erroneously attracted to nearby arteries or other rectilinear structures. On the other hand, the absence of nearby attractors would lead to the loss of local deformation information due to the lack of image forces at the distal points; the distal segments would then be subjected to only the globally recovered motion of the coronary tree. In general, however, these distal parts of the coronaries are not important for interventional purposes, but the use of temporally continuous motion models may help overcome their periodic disappearance.

The quality of the motion tracking algorithm is ultimately tied to the quality of the algorithm used to detect vessels in the angiograms. As described in Section II-C, a multiscale method based on graylevel second derivatives is used to automatically detect rectilinear structures in the images [24]. As seen in Fig. 1(b), the detection of proximal segments is somewhat suppressed. This may be the result of the range of scales, $\sigma=\{1,2,3,4,5\}$ pixels, used for the clinical validation procedure. The detection of the larger proximal vessels would be improved with the use of larger scales of detection. However, at larger scales, the erroneous detection of background structures such as ribs, vertebrae, and the diaphragm, and the merging of neighboring small vessels becomes more problematic. In practice, the set of filter sizes used for each patient should be optimized for the given imaging conditions, or an alternative vessel detection method could be explored.

\section{Future DeVElopments}

Dynamic 3-D models of the coronary arteries are needed for various applications in addition to their conventional uses for cardiovascular and coronary disease diagnosis as presented in Section I. For example, the use of robots in facilitating surgical procedures is an active field of study [33]-[35]. Minimally invasive procedures, which are performed through a few small incisions, are a particular focus of attention. The limited field of view, and the remote point of action of the laparoscopic instruments, generate unique challenges for the surgeon. In minimally invasive coronary artery bypass procedures, the cardiac motion, and the enveloping pericardium complicate the task of localizing the arterial segment targeted for intervention. Intraoperative registration of preoperative reconstructions of the coronaries is being studied as an augmented reality tool to aid the surgeon.

As an alternative to X-ray angiography, magnetic resonance coronary angiography (MRCA) is being actively investigated for use as a noninvasive diagnostic and screening tool for coronary artery disease [36]. However, because of the noninstantaneous nature of the MR image acquisition, images of the coronary arteries are degraded by cardiac and respiratory motion. A comprehensive analysis of the coronary motion is necessary for development of motion gating and correction techniques for improvement of MRCA. A preliminary 2-D study of the rest period of the coronaries during the cardiac cycle has been presented in [37]. Respiratory motion has been studied using MR line scanning techniques [38] and fast multi-slice 2-D acquisitions [39]. The 3-D motion tracking method presented in this paper can be used to provide a previously unseen comprehensive 3-D motion and deformation map from high temporal and spatial resolution images of the coronaries.

\section{CONCLUSION}

In this paper, a 3-D method for tracking the motion of coronary arteries in a biplane angiogram sequence has been presented. A registration framework recovers the motion one cine phase at a time, using a set of coarse-to-fine motion models. This 3-D approach provides a spatially coherent method for tracking the arteries through projective occlusions, and allows the use of true 3-D regularizing constraints on the temporal evolution of the vessels. Finally, efficacy of this method has been demonstrated by successfully tracking the left coronary tree over one cardiac cycle in five patient image sets.

\section{ACKNOWLEDGMENT}

The authors would like to thank Dr. K. Abdul-Nour, B. Schenke, S. White, G. Zalos, and other members of the Cardiac Catheterization Section of the National Heart Lung and Blood Institute, $\mathrm{NIH}$, for their assistance in obtaining patient data, J. Lee for construction of the calibration grid and phantom, and Dr. A. Dick and Dr. R. Lederman for assistance in anatomical labeling. They extend special thanks to $\mathrm{C}$. Blondel and $\mathrm{H}$. Delingette for numerous discussions and helpful suggestions. 


\section{REFERENCES}

[1] A. Wahle, E. Wellnhofer, I. Mugaragu, H. Sauer, H. Oswald, and E. Fleck, "Assessment of diffuse coronary artery disease by quantitative analysis of coronary morphology based upon 3-D reconstruction from biplane angiograms," IEEE Trans. Med. Imag., vol. 14, pp. 230-241, June 1995.

[2] T. Saito, M. Misaki, K. Shirato, and T. Takishima, "Three-dimensional quantitative coronary angiography," IEEE Trans. Biomed. Eng., vol. 37, pp. 768-777, Aug. 1990.

[3] P. Windyga, M. Garreau, H. LeBreton, and J. Coatrieux, "2-D and 3-D knowledge combination for the reconstruction of coronary arteries: First results on real data," in Proc. IEEE Eng. Med. Biol. Soc., vol. 1, 1994, pp. 602-621.

[4] H. Wollschlager, P. Lee, A. Zeiher, U. Solzbach, T. Bonzel, and H. Just, "Derivation of spatial information from biplane multidirectional coronary angiograms," Med. Prog. Technol., vol. 611, no. 2, pp. 57-63, 1986.

[5] S.-Y. James Chen and C. Metz, "Improved determination of biplane imaging geometry from two projection images and its application to three-dimensional reconstruction of coronary arterial trees," Med. Phys., vol. 24, no. 5, pp. 633-654, May 1997.

[6] F. Mourgues, F. Devernay, G. Malandain, and E. Coste-Manière, "3D+t modeling of coronary artery tree from standard non simultaneous angiograms," in Proc. MICCAI, vol. 2208, Oct. 2001, pp. 1320-1322.

[7] A. Young, P. Hunter, and B. Smaill, "Estimation of epicardial strain using the motions of coronary bifurcations in biplane cinéangiography," IEEE Trans. Biomed. Eng., vol. 39, pp. 526-531, May 1992.

[8] G. Coppini, M. Demi, P. Marraccini, and A. L'Abbate, "3-D heart motion from X-ray angiography," Comput. Cardiol., pp. 71-74, 1995.

[9] J. Puentes, C. Roux, M. Garreau, and J. Coatrieux, "Dynamic feature extraction of coronary artery motion using DSA image sequences," IEEE Trans. Med. Imag., vol. 17, pp. 857-871, Dec. 1998.

[10] N. Guggenheim, P. Dorsaz, P. Doriot, C. Suilen, F. Chappuis, and W. Rutishauser, "3-D determination of the intravascular volume and flow of coronary arteries," Int. J. Biomed. Comput., vol. 35, pp. 13-23, 1994.

[11] J. Meunier, M. Bourassa, M. Bertrand, M. Verreault, and G. Mailloux, "Regional epicardial dynamics computed from coronary cineangiograms," Comput. Cardiol., pp. 307-310, 1989.

[12] B. Tom, S. Efstratiadis, and A. Katsaggelos, "Motion estimation of skeletonized angiographic images using elastic registration," IEEE Trans. Med. Imag., vol. 13, pp. 450-460, Sept. 1994.

[13] R. Curwen, A. Amini, J. Duncan, and F. Lee, "Tracking vascular motion in X-ray image sequences with Kalman snakes," Comput. Cardiol., pp. 109-112, 1994

[14] M.-P. Dubuisson-Jolly, C. Liang, and A. Gupta, "Optimal polyline tracking for artery motion compensation in coronary angiography," in Proc. ICCV, 1998, pp. 414-419.

[15] S. Ruan, A. Bruno, R. Collorec, and J. Coatrieux, "3-D motion and reconstruction of coronary networks," in Proc. IEEE EMBS, vol. 5, 1992, pp. 2048-2049.

[16] B. Bascle and R. Deriche, "Stereo matching, reconstruction and refinement of 3-D curves using deformable contours," in Proc. ICCV, 1993, pp. 421-430.

[17] P. Radeva, R. Toledo, C. Von Land, and J. Villanueva, "3-D vessel reconstruction from biplane angiograms using snakes," Comput. Cardiol., vol. 25, pp. 773-776, 1998.

[18] C. Cañero, P. Radeva, R. Toledo, J. Villanueva, and J. Mauri, "3-D curve reconstrucion by biplane snakes," in Proc. ICPR, vol. 4, 2000, pp. 563-566.

[19] R. Toledo, P. Radeva, C. Von Land, and J. Villanueva, "3-D dynamic model of the coronary tree," Comput. Cardiol., vol. 25, pp. 777-780, 1998.

[20] R. Toledo, X. Orriols, P. Radeva, X. Binefa, J. Vitrià, C. Cañero, and J. Villanueva, "Eigensnakes for vessel segmentation in angiography," in Proc. ICPR, vol. 4, 2000, pp. 340-343.
[21] L. Sarry and J.-Y. Boire, "Three-dimensional tracking of coronary arteries from biplane angiographic sequences using parameterically deformable models," IEEE Trans. Med. Imag., vol. 20, pp. 1341-1351, Dec 2001.

[22] W. Baxter and A. McCulloch, "In vivo finite element model-based image analysis of pacemaker lead mechanics," Med. Image Anal., vol. 5, pp. 255-270, 2001.

[23] G. Shechter, F. Devernay, E. Coste-Maniere, and E. McVeigh, "Temporal tracking of 3-D coronary arteries in projection angiograms," Proc. SPIE Med. Imag., vol. 4684, pp. 612-623, 2002.

[24] A. Frangi, W. Niessen, R. Hoogeveen, T. van Walsum, and M. Viergever, "Model-based quantitation of 3-D magnetic resonance angiographic images," IEEE Trans. Med. Imag., vol. 18, pp. 946-956, Oct 1999.

[25] L. Piegl and W. Tiller, The NURBS Book, 2nd ed. Berlin, Germany: Springer-Verlag, 1997.

[26] J. Declerck, J. Feldmar, M. Goris, and F. Betting, "Automatic registration and alignment on a template of cardiac stress and rest reoriented SPECT images," IEEE Trans. Med. Imag., vol. 16, pp. 727-737, Dec. 1997.

[27] P. Radeva, A. Amini, and J. Huang, "Deformable B-solids and implicit snakes for 3-D localization and tracking of SPAMM MRI data," Comput. Vis. Image Understanding, vol. 66, no. 2, pp. 163-178, May 1997.

[28] J. Montagnat, H. Delingette, N. Scapel, and N. Ayache, "Representation, shape, topology and evolution of deformable surfaces. Applications to 3-D medical image segmentation,” INRIA, Paris, France, Tech. Rep. 3954, May 2000

[29] E. Wellnhofer, A. Wahle, I. Mugaragu, J. Gross, H. Oswald, and E. Fleck, "Validation of an accurate method for three-dimensional reconstruction and quantitative assessment of volumes, lengths and diameters of coronary vascular branches and segments from biplane angiographic projections," Int. J. Card. Imag., vol. 15, no. 5, pp. 339-353, Oct. 1999

[30] R. Berne and M. Levy, Cardiovascular Physiology, 7th ed. St. Louis, MO: Mosby, 1997, ch. 10, p. 225.

[31] G. Shechter, C. Ozturk, and E. McVeigh, "Interactive four-dimensional segmentation of multiple image sets," Proc. SPIE, vol. 3976, pp. $165-173,2000$.

[32] C. Ozturk and E. McVeigh, "Four dimensional B-spline based motion analysis of tagged cardiac MR images," Proc. SPIE Med. Imag., vol. 3660, pp. 46-56, Feb. 1999.

[33] B. Davies, "A review of robotics in surgery," in Proc. Inst. Mech. Engrs. vol. 214, 2000, pp. 129-140.

[34] R. Howe and Y. Matsuoka, "Robotics for surgery," Annu. Rev. Biomed Eng., vol. 1, pp. 211-240, 1999.

[35] S. Jacobs and V. Falk, "Pearls and pitfalls: Lessons learned in endoscopic robotic surgery-the da Vinci experience," Heart Surg. Forum, vol. 4, no. 4, pp. 307-310, 2001

[36] W. Yong Kim, P. Danias, M. Stuber, S. Flamm, S. Plein, E. Nagel, S. Langerak, O. Weber, E. Pedersen, M. Schmidt, R. Botnar, and W. Manning, "Coronary magnetic resonance angiography for the detection of coronary stenoses," N. Eng. J. Med., vol. 345, no. 26, pp. 1863-1869, Dec. 2001.

[37] Y. Wang, E. Vidan, and G. Bergman, "Cardiac motion of coronary arteries: Variability in the rest period and implications for coronary MR angiography," Radiology, vol. 213, pp. 751-758, 1999.

[38] A. Holland, J. Goldfarb, and R. Edelman, "Diaphragmatic and cardiac motion during suspended breathing: Preliminary experience and implications for breath-hold MR imaging," Radiology, vol. 209, no. 2, pp. 483-489, Nov. 1998.

[39] Y. Wang, S. Riederer, and R. Ehman, "Respiratory motion of the heart: Kinematics and the implications for the spatial resolution in coronary imaging," Magn. Reson. Med., vol. 33, no. 5, pp. 713-719, May 1995. 